\title{
Dielectric Relaxations in Poly( $\gamma$-n-alkyl L-glutamate)s II. Study of Relaxation due to Motion of the Side Chain and that due to Motion of the Main Chain
}

\author{
Maeko KaKIZAKI, Hirofumi NAKAYAma, and Teruo Hideshima
}

Department of Applied Physics, Faculty of Engineering, Hokkaido University, Sapporo 060, Japan

(Received July 25, 1985)

\begin{abstract}
In order to get detailed information about the $\beta$-relaxation due to motion of side chains in poly $(\gamma$-methyl L-glutamate), poly( $\gamma$-ethyl L-glutamate) and poly $(\gamma$ - $n$-propyl L-glutamate), the frequency and temperature dependences of loss permittivity were determined in the frequency range from $10^{-4} \mathrm{~Hz}$ to $10^{5} \mathrm{~Hz}$ and at temperatures ranging from $c a .-100^{\circ} \mathrm{C}$ to $c a .60^{\circ} \mathrm{C}$. The shape of the loss permittivity as a function of frequency was independent of the side-chain length. The loci of loss-permittivity peaks on the relaxation map could be represented by two straight lines independent of the side-chain length. They intersect in the glass transition region associated with the $\alpha$-relaxation due to the motion of the main chain. The activation energy below the glass transition region was thought to be essentially due to side-chain motion and estimated to be $c a$. $18 \mathrm{kcal} \mathrm{mol}^{-1}$. The value of the activation energy and shape of the loss permittivity curve are very similar to those of the $\beta$-relaxation in poly ( $n$-alkyl methacrylate)s. The two-site model analysis of the relaxation strength also showed similarity. On the basis of these results, the molecular mechanism of the $\alpha$-relaxation and the nature of the glass transition are explained in terms of the motion of the $\alpha$-helix.
\end{abstract}

KEY WORDS Dielectric Relaxation / Loss Permittivity / Side Chain / Glass Transition / Two-Site Model / Rotational Relaxation / Rod-like Polymer / Poly( $\gamma$-methyl L-glutamate) / Poly( $\gamma$-ethyl L-glutamate) / Poly( $\gamma$ - $n$-propyl Lglutamate) /

Poly $(\gamma$ - $n$-alkyl L-glutamate)s, (PnALG)s, are a series of polymers belonging to synthetic polypeptides, whose structures and properties in solid state have been studied recently by several investigators in some detail. ${ }^{1-10}$ They are characterized by flexible long side chains which are chemically quite similar to those of poly(n-alkyl methacrylate)s, (PnAMA)s, a well-known series of amorphous polymers. However, the conformations of the main chains of these two series of polymers are very different from each other; the main-chain conformation of (PnALG)s is usually the $\alpha$-helix which is rather rigid with intrachain hydrogen bonds, while that of (PnAMA)s is usually a random coil. Further, the helices of (PnALG)s in solid state have a hexagonal arrangement with an amorphous phase of the side chains around them, ${ }^{8}$ whereas the random coils of (PnAMA)s are in a typically amorphous phase together with the side chains.

According to investigations reported so far, the dielectric and mechanical measurements of these two series of polymers reveal three relaxations named $\alpha, \beta$ and $\gamma$ in the order of descending temperature. In the case of (PnAMA)s, these relaxations have been attributed to the motions of the main chain, the side chain as a whole, and a part of the side chain, respectively. ${ }^{11}$ As for (PnALG)s, it is thought that the relation between the molecular motions and the relaxations is similar to that in the case of (PnAMA)s.

However, several authors have pointed out 
that there is an essential difference between the $\beta$-relaxation in (PnALG)s and that in (PnAMA)s, ${ }^{3,6,9,10}$ though the modes of molecular motion of the side chains in these two series of polymers are similar to each other. According to them, temperature dependence of the dielectric and mechanical relaxation times for the $\beta$-relaxation in (PnALG)s can be represented by the WLF equation and consequently, the motion of the side chain responsible for the $\beta$-relaxation should be frozen at a certain temperature like the glass transition of the micro-Brownian motion of the main chain in amorphous polymers. They also found a break point in the temperature dependence of specific volume for $\operatorname{poly}(\gamma$-methyl L-glutamate) at $c a .-8^{\circ} \mathrm{C}$ and regarded it as the glass transition temperature for the sidechain motion.?

In a previous paper, ${ }^{12}$ we reported that the dielectric $\alpha$-relaxation found in (PnALG)s has the relaxation time obeying the WLF equation and reveals the glass transition of the underlying molecular motion. As discussed in the previous paper, this glass transition should be caused by the freezing of some mode of motion of the main chain. Consequently, it seems important to clarify the relation between the glass transition associated with the $\alpha$-process and that associated with the $\beta$-process.

In the present paper, we investigate the relaxational behavior of the $\beta$-process in a wide range of frequencies and temperatures above and below the glass transition temperature for the $\alpha$-process to clarify the effects of the transition on the $\beta$-relaxation and ascertain the existence of the glass transition of the sidechain motion. Further, the nature of the $\alpha$ relaxation and the glass transition associated with the motion of the main chain is considered in connection with the nature of the $\beta$ relaxation.

\section{EXPERIMENTAL}

\section{Samples}

The samples were methyl, ethyl and $n$ propyl glutamate polymers, the same as those used in the investigation of the $\alpha$-relaxation in the previous paper ${ }^{12}$ and designated as PMLG, PELG, and PnPLG respectively. The method of casting a film from a chloroform solution and the procedure of heat treatment of the film were also the same as reported before.

\section{Measurements}

The loss permittivity in the frequency range from $10^{-4} \mathrm{~Hz}$ to $10^{-2} \mathrm{~Hz}$ was determined from absorption current measured with a vibratingreed electrometer made by Takeda Riken Industry Co., as in the previous paper, ${ }^{12}$ while the loss and storage permittivities from $10^{-1} \mathrm{~Hz}$ to $10 \mathrm{~Hz}$ and from $30 \mathrm{~Hz}$ to $100 \mathrm{kHz}$ were measured with the two types of bridge apparatus, TR-4 and TR-10, made by Ando Electric Co. respectively. The measurements were mainly made from $c a .-100^{\circ} \mathrm{C}$ to $c a$. $60^{\circ} \mathrm{C}$. In order to get the overall loss pattern, temperature dependence of loss permittivity in the range from $c a .-190^{\circ} \mathrm{C}$ to $c a .170^{\circ} \mathrm{C}$ was measured at $30 \mathrm{~Hz}$ especially for PMLG. Temperature was determined with a copperconstantan thermocouple placed near the sample and its fluctuation during the measurements was kept within $\pm 0.5^{\circ} \mathrm{C}$. The measurements were mainly made in the low temperature range, because the data on the $\beta$-relaxation at low temperatures are rather scarce in the literature.

\section{RESULTS AND DISCUSSION}

\section{The $\beta$-Relaxation}

Figure 1 shows the temperature dependence of loss permittivity measured at $30 \mathrm{~Hz}$ for PMLG. There are three relaxations in which the $\alpha$-relaxation was investigated in the previous paper. The overall loss pattern of the 


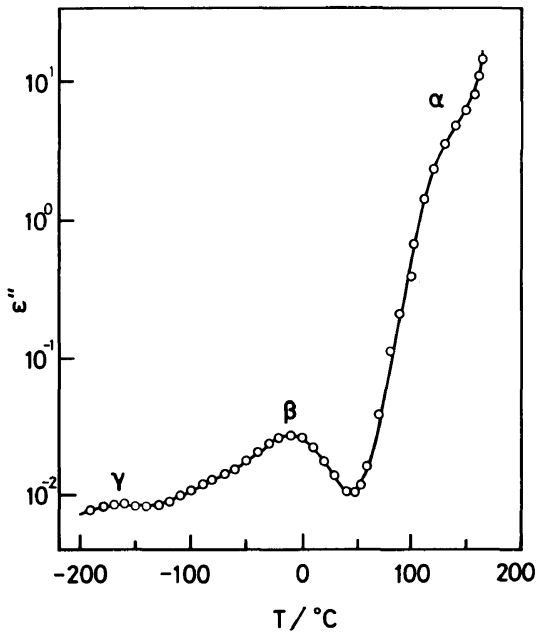

Figure 1. Temperature dependence of loss permittivity for PMLG at $30 \mathrm{~Hz}$.

other two samples is quite similar to that of PMLG. Frequency dependences of storage and loss permittivities for the $\beta$-relaxation in PMLG at various temperatures are given in Figures 2 and 3. For this sample, the measurement of absorption current was also made but the data are not used here, because these were rather uncertain.

On the assumption of the time-temperature reducibility, ${ }^{13}$ the master curves reduced to $-30^{\circ} \mathrm{C}$ could be constructed using the horizontal shift with the slight vertical shift and are shown in Figures 4 and 5, respectively. For the reduction of the data on storage permittivity, the high-frequency limit, $\varepsilon_{\infty}$, was assumed to be independent of temperature. Its value was estimated to be 2.96 from the low-temperature data on storage permittivity measured at $3 \mathrm{~Hz}$. In Figure 4 , the loss permittivity obtained by the approximate relation, ${ }^{14} \varepsilon^{\prime \prime}=-\pi / 4.6\left(\mathrm{~d} \varepsilon^{\prime} /\right.$ $\mathrm{d} \log \omega)$, is also shown. Here $\omega$ is angular frequency. The shape of this curve is in fair agreement with that of the loss permittivity master curve on a doubly logarithmic plot.

The vertical shift factor, $C_{\mathrm{T}}$, as the ratio of relaxation strength to that at the reference temperature, $-30^{\circ} \mathrm{C}$, was obtained from the data in Figure 3 and is shown in Figure 6 as a

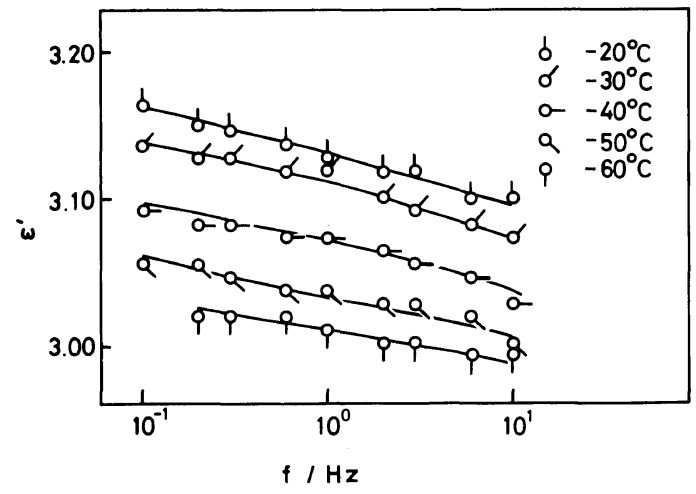

Figure 2. Frequency dependence of storage permittivity for PMLG at the indicated temperatures.

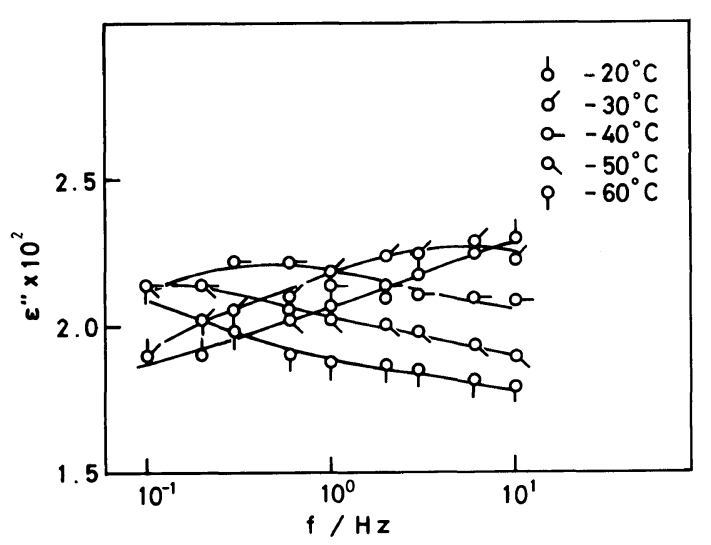

Figure 3. Frequency dependence of loss permittivity for PMLG at the indicated temperatures.

function of reciprocal absolute temperature. The values of $\log C_{\mathrm{T}}$ at $-50^{\circ} \mathrm{C}$ and $-40^{\circ} \mathrm{C}$ were determined by fitting the double logarithmic plots of the loss-permittivity data at these temperatures to those at $-30^{\circ} \mathrm{C}$. As these values are represented by a straight line in Figure 6, the extrapolation of the straight line was used to estimate the values in this temperature range.

The horizontal shift factors for loss and storage permittivities are also shown in Figure 6 . They agree very well with each other and fall on a straight line. This fact and the agreement of the master curves from loss and storage permittivities assure the consistency of the method of reduced variables. The activation 


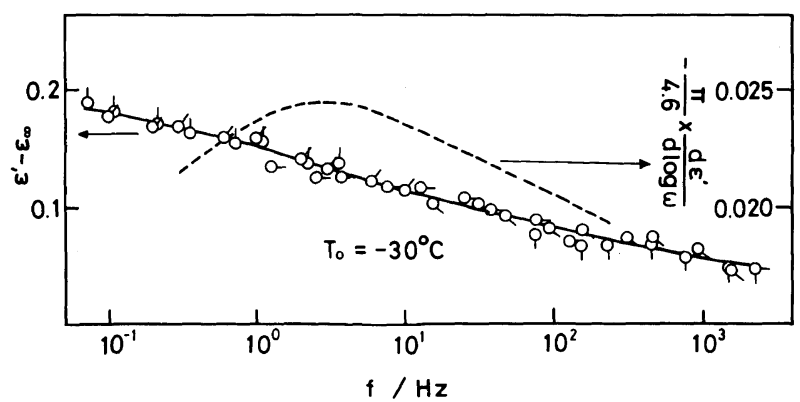

Figure 4. Master curve of storage permittivity and approximate loss permittivity derived from it for the $\beta$-relaxation in PMLG reduced to the reference temperature $T_{\mathrm{o}}=-30^{\circ} \mathrm{C}$. The high-frequency limit of storage permittivity, $\varepsilon_{\infty}$, is assumed to be independent of temperature and its value is estimated to be 2.96 . The temperature key is the same as in Figure 2.

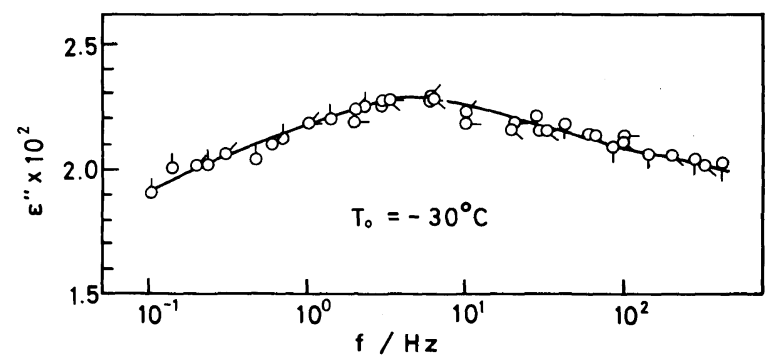

Figure 5. Master curve of loss permittivity for the $\beta$-relaxation in PMLG reduced to the reference temperature $T_{\mathrm{o}}=-30^{\circ} \mathrm{C}$. The temperature key is the same as in Figure 3 .

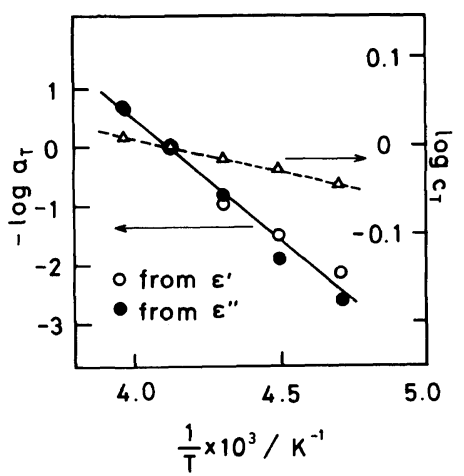

Figure 6. Horizontal shift factor $\mathrm{a}_{\mathrm{T}}$ and vertical shift factor $C_{\mathrm{T}}$ for the $\beta$-relaxation in PMLG reduced to $-30^{\circ} \mathrm{C}$ and plotted against reciprocal absolute temperature. Open and closed circles, for the horizontal shift factors obtained from storage permittivity and loss permittivity respectively; open triangles, for the vertical shift factor obtained from temperature dependence of the loss permittivity maximum.

energy estimated from the slope of the straight line in Figure 6 is $c a .19 \mathrm{kcal} \mathrm{mol}^{-1}$.
As for PELG and PnPLG, the data on the time dependence of absorption current, $I_{\mathrm{d}}$, in the $\beta$-relaxation region were measured at various temperatures. The results could be represented by the power function of time as $I_{\mathrm{d}} \propto t^{-n}$ and the value of $n$ satisfied the condition for the Hamon approximation, ${ }^{15} 0.3<n<1.2$. Consequently, the loss permittivity, $\varepsilon^{\prime \prime}$, of these samples was obtained from $I_{\mathrm{d}}$ as a function of frequency, $f$, or angular frequency, $\omega$, by means of the Hamon approximation,

$$
\begin{aligned}
& \varepsilon^{\prime \prime}(\omega)=I_{\mathrm{d}}(t) / \omega C_{0} V=t \phi(t) / 0.63 \\
& t=0.63 / \omega=0.1 / f
\end{aligned}
$$

where $C_{0}$ and $V$ denote the capacitance of the electrodes without the sample and the applied step voltage respectively, while $\phi(t)$ denotes the decay function or the after-effect function.

In Figures 7 and 8, frequency dependences of loss permittivity for PELG and PnPLG obtained by means of eq 1 and 2 are shown at 


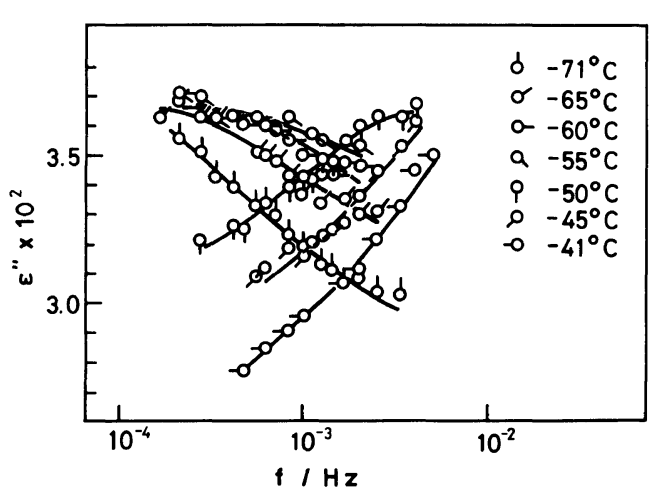

Figure 7. Frequency dependence of loss permittivity for PELG calculated from absorption current at the indicated temperatures. Frequency $f$ corresponds to time $t$ through the Hamon approximation, $f=0.1 / t$.

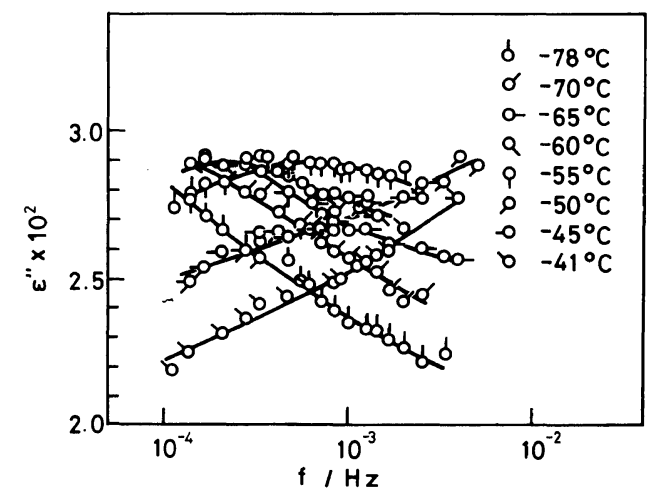

Figure 8. Frequency dependence of loss permittivity for PnPLG calculated from absorption current at the indicated temperatures. Frequency $f$ corresponds to time $t$ through the Hamon approximation, $f=0.1 / t$.

various temperatures, respectively. From these data, the master curves could be constructed by the horizontal shift alone. Consequently, the vertical shift in this temperature range, if any, should be very small. The master curves are shown in Figures 9 and 10, while the horizontal shift factors are plotted against the reciprocal absolute temperature in Figure 11. As the shift factors for these two samples can be represented by the same straight line, the activation energies are nearly equal to each other. The activation energy obtained from the slope of the straight line is $c a .18 \mathrm{kcal} \mathrm{mol}^{-1}$.

From the results mentioned above, it should be noted that the horizontal shift factors for these three samples can be represented by the Arrhenius equation as a function of the reciprocal absolute temperature in this temperature range and the activation energies are nearly equal to each other and to that for the $\beta$-relaxation in (PnAMA)s. ${ }^{16}$

The frequency-temperature positions of the $\alpha$-and $\beta$-relaxations in (PnALG)s are shown in the relaxation map in Figure 12. The reciprocals of the shift factors for the $\alpha$-relaxation in the three samples reported in the previous paper ${ }^{12}$ are plotted through the peak positions in this figure to give the loci of the loss permittivity maxima. The temperature dependence of these curves is represented by the WLF equation and the temperature coefficients of the curves are appreciably reduced at and below the glass transition temperatures, which are $5^{\circ} \mathrm{C},-4^{\circ} \mathrm{C}$, and $-7^{\circ} \mathrm{C}$ for PMLG, PELG, and PnPLG, respectively, as shown in the previous paper.

As for the $\beta$-relaxation, the positions of the loss permittivity peaks in the audiofrequency region were determined for PMLG and PELG, while those in the ultralow-frequency region were determined for the three samples. In both of these frequency regions, the peak positions are almost independent of the side-chain length and can be represented by straight lines which intersect each other near the glass transition temperatures for the $\alpha$-process.

On the other hand, the shift factors for the $\beta$-relaxation given in Figures 6 and 11 can be represented also by a straight line, independent of the side-chain length when these are plotted through the loss permittivity maxima on the relaxation map. The straight line thus obtained is parallel to the straight line representing the data at ultralow frequencies and in a frequency region somewhat lower than that in which the latter lies. This shift of the data along the frequency axis is thought to be due to differences in measuring methods and thermal history of the sample. In Figure 12, the data on the shift factors have been moved upward 


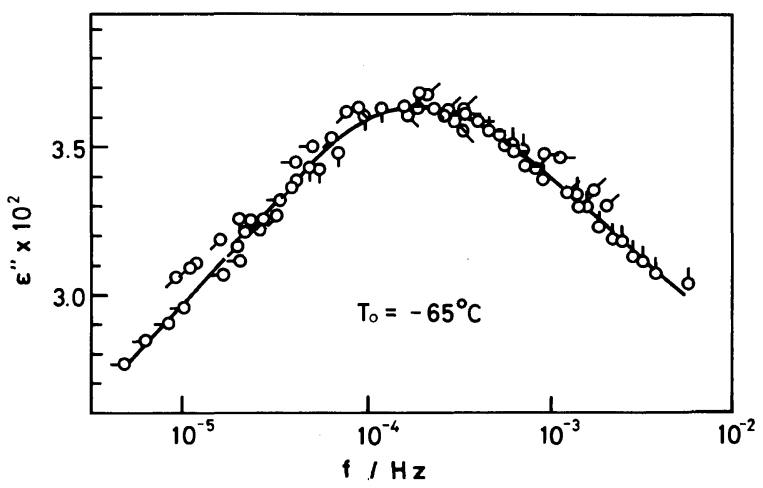

Figure 9. Master curve of loss permittivity for the $\beta$-relaxation in PELG reduced to the reference temperature $T_{\mathrm{o}}=-65^{\circ} \mathrm{C}$, obtained from the data on absorption current. The temperature key is the same as in Figure 7.

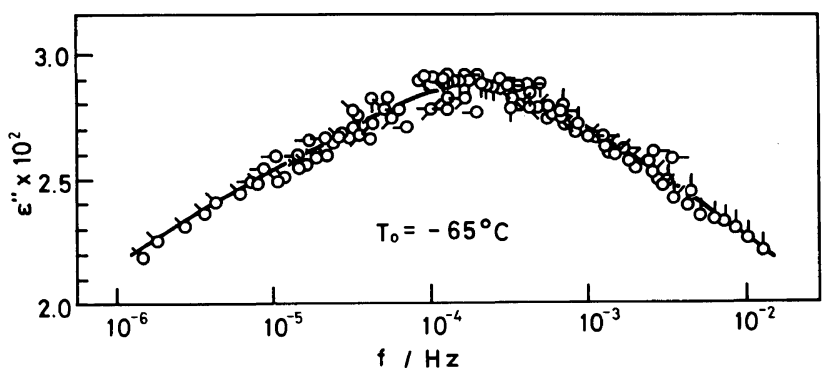

Figure 10. Master curve of loss permittivity for the $\beta$-relaxation in PnPLG reduced to the reference temperature $T_{\mathrm{o}}=-65^{\circ} \mathrm{C}$, obtained from the data on absorption current. The temperature key is the same as in Figure 8.

from the original positions in such a way that the straight line representing them coincides with that representing the data at ultralow frequencies.

As shown in Figure 12, the positions of the loss permittivity maxima for the $\beta$-relaxation in the three samples can be represented by the two straight lines intersecting near the glass transition temperatures for the $\alpha$-process. The activation energy below the glass transition temperatures is $c a$. $18 \mathrm{kcal} \mathrm{mol}^{-1}$, while that above the glass transition temperatures is $c a$. $22 \mathrm{kcal}$ mol. A similar change in the slope of the straight line of the Arrhenius plot near the glass transition temperature was found in the case of the dielectric $\beta$-relaxation in polymethyl and polyethyl methacrylates. ${ }^{17}$ The value of the activation energy above the glass transition temperature is thought to be modified by motion of the main chain. ${ }^{17}$

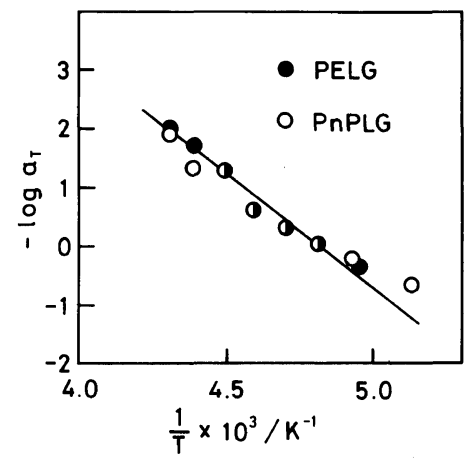

Figure 11. Horizontal shift factor $\mathrm{a}_{\mathrm{T}}$ for the $\beta$-relaxation in PELG and PnPLG reduced to $-65^{\circ} \mathrm{C}$ and plotted against reciprocal absolute temperature. Closed circles, for PELG; open circles, for PnPLG. Half-closed circles denote open and closed circles on the same position. 


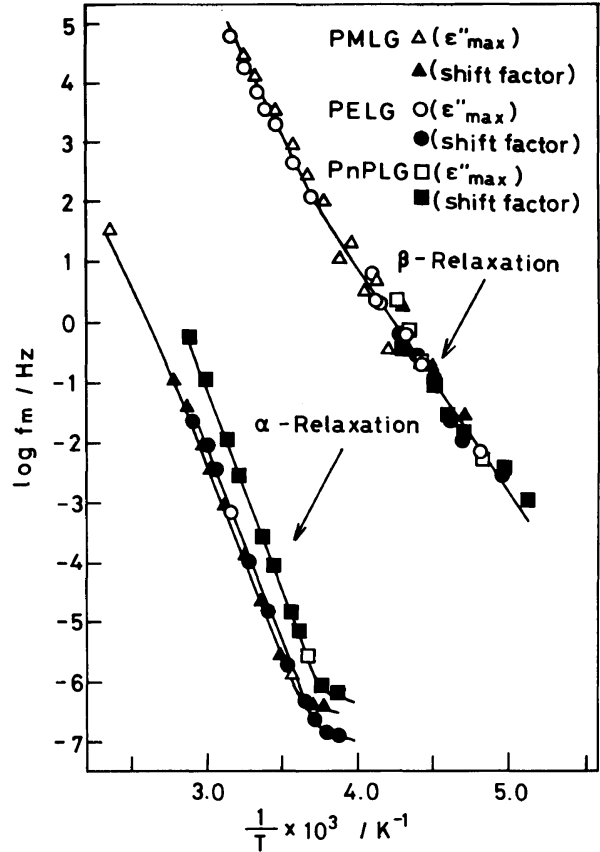

Figure 12. Plots of log (frequency) against reciprocal absolute temperature for the loss-permittivity maxima of the $\alpha$ - and $\beta$-relaxations in PMLG (triangles), PELG (circles) and PaPLG (squares). Open symbols denote the positions of the loss-permittivity maxima; closed symbols denote the positions of the reciprocals of the shift factors plotted through the positions of loss permittivity maxima.

It is remarkable that the locus of the $\beta$-loss peak for PMLG in Figure 12 extends to temperatures far below $-8^{\circ} \mathrm{C}$, often regarded as the glass transition temperature for the motion of the side chain as a whole or the motion of the side chain responsible for the $\beta$-relaxation. This fact means that such motion of the side chain is not frozen at this temperature or at any temperature investigated here. The loci of the $\beta$-loss peaks for PELG and PnPLG also do not reveal any manifestation of freezing throughout the entire temperature range.

Further, the shape of the loss permittivity curves for the $\beta$-relaxation observed in the high-temperature region is nearly the same as that observed in the low-temperature region, though the width becomes a little broader below the glass transition temperature. Figure

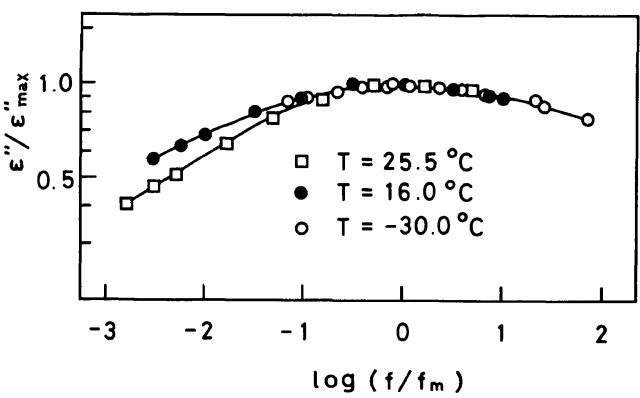

Figure 13. Normalized plots of loss permittivity for the $\beta$-relaxation in PMLG at the indicated temperatures. Here, $f_{\mathrm{m}}$ is the frequency for the loss-permittivity maximum, $\varepsilon_{\max }^{\prime \prime}$.

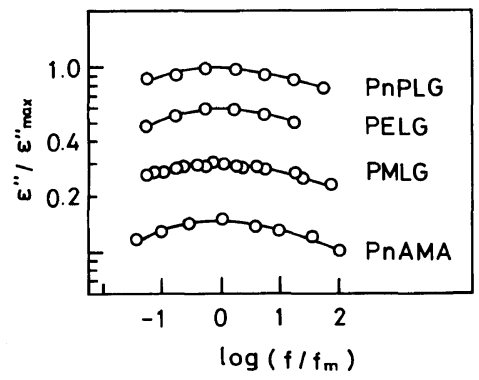

Figure 14. Normalized plots of loss permittivity for the $\beta$-relaxation in PMLG, PELG, PnPLG and poly $(n-$ alkyl methacrylate). Here, $f_{\mathrm{m}}$ is the frequency for the loss-permittivity maximum, $\varepsilon_{\max }^{\prime \prime}$. The ordinate scale is correct for the curve of PnPLG. The other curves have shifted downward successively.

13 shows the case of PMLG as an example. This also assures that the mechanism of the $\beta$ relaxation does not change essentially in this temperature range. Consequently, the glass transition occurs only in the case of the molecular motion responsible for the $\alpha$-relaxation in this temperature range.

Figure 14 compares the shapes of the loss permittivity curves for the $\beta$-relaxation in the three samples in a normalized form. The data on PMLG, PELG and PnPLG are the same as given in Figures 5, 9 and 10. It is evident that the shape of the curve on the doubly logarithmic plot is independent of the side-chain length as in the case of the $\beta$-relaxation in (PnAMA)s. ${ }^{16}$ Moreover, the shape of the loss permittivity curve for the $\beta$-relaxation in 
(PnALG)s is quite similar to that in (PnAMA)s reported in the previous paper $^{16}$ as shown in Figure 14.

\section{The Two-Site Model}

Further similarity between (PnALG)s and (PnAMA)s was found when the molecular process of the $\beta$-relaxation was analyzed by means of the two-site model. For this model, the relaxation strength $\Delta \varepsilon$ associated with the dipole reorientation between the two stable states is given by the relation, ${ }^{18}$

$$
\Delta \varepsilon=\frac{4 \pi N(\Delta \mu)^{2}}{3 k T}\left(\frac{\varepsilon_{\infty}+2}{3}\right)^{2}\left(\frac{3 \varepsilon_{0}}{2 \varepsilon_{0}+\varepsilon_{\infty}}\right) \frac{K}{(1+K)^{2}}
$$

where $N$ is the number of dipoles per unit volume, $\Delta \mu$ is the difference between the dipole moments at the two states, $K=\exp (\Delta S / k) \times$ $\exp (-\Delta H / k T)$ is the equilibrium constant with $\Delta S$ and $\Delta H$ being the entropy and enthalpy differences between the two states, and $\varepsilon_{0}$ and $\varepsilon_{\infty}$ are the low- and high-frequency limits of storage permittivity.

If $K$ is much smaller than unity in Eq 3, $\log (T \Delta \varepsilon)$ plotted against reciprocal absolute temperature should give a straight line. Figure 15 shows this relation in the case of PMLG. Here the value of $\Delta \varepsilon$ at $-30^{\circ} \mathrm{C}$ was estimated to be 0.2 assuming that $\varepsilon_{0}$ was given by the extrapolation of the straight line representing the stationary region of storage permittivity at high temperatures measured at $3 \mathrm{~Hz}$ and that $\varepsilon_{\infty}$ was 2.96 as in Figure 4 . The temperature coefficient of $\Delta \varepsilon$ given as the vertical shift factor in Figure 6 was used to calculate the values of $\Delta \varepsilon$ at other temperatures. The value of $\Delta H$ determined from the slope of the straight line is $c a$. $0.8 \mathrm{kcal} \mathrm{mol}^{-1}$, which is close to $0.7 \mathrm{kcal} \mathrm{mol}^{-1}$ for poly(methyl methacrylate). ${ }^{16}$ Therefore, the elementary molecular processes in these polymers seem to be similar to each other, though the origin of the relaxation spectrum is unknown.

From these facts and the similarity in the values of activation energy, it can be con-

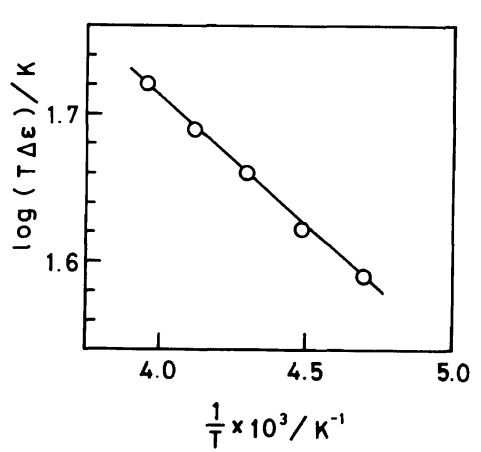

Figure 15. Plot of $\log (T \Delta \varepsilon)$ for the $\beta$-relaxation in PMLG against the reciprocal absolute temperature.

cluded that the $\beta$-relaxations in these two series of polymers are caused by similar molecular processes of the side chains irrespective of differences in the conformation and chemical structures of the main chains.

\section{The Nature of the $\alpha$-Relaxation}

The remaining problems are concerned with the molecular process of the $\alpha$-relaxation and the nature of the glass transition in (PnALG)s. As discussed in the previous paper, ${ }^{12}$ the molecular process for the dielectric $\alpha$-relaxation seems to be responsible for the mechanical one as well. The $\alpha$-relaxation might be caused by the molecular motion of the main chain in a disordered state. If this is the case, the glass transition associated with the $\alpha$-relaxation should be due to freezing of the motion of the main chain in the disordered state. However, it seems doubtful that the number of such disordered main chains would always be enough to make the $\alpha$-relaxation so prominent, as shown in Figure 1.

A possible explanation is given by considering the reorientation of the rigid $\alpha$-helix. The $\alpha$ helix has a large dipole moment along the helical axis and is surrounded by flexible side chains. These side chains are expected to function as a viscous liquid, slowing the motion of the rigid $\alpha$-helix in an electric field, because the $\beta$-process due to the internal motion of the side chain should have already relaxed at the tem- 
perature and frequency for the $\alpha$-relaxation. In this case, the situation can be represented by a simple model which consists of polar rod-like molecules in a hexagonal arrangement with a viscous liquid among them.

The rigid $\alpha$-helix may execute various modes of motion, such as translation in the direction of the helical axis, rotation about the helical axis and rotation about the axis perpendicular to the helical axis, though these are highly restricted. However, only the last mode given above can be responsible for the orientation polarization of the $\alpha$-helix. In addition, the helical axis is thought to have a tendency to lie parallel to the film surface. Therefore, we calculated the relaxation time and relaxation strength of the $\alpha$-relaxation using this model. The $\beta$-relaxation corresponds to the relaxation due to some internal motion of the liquid molecule in this model.

According to recent studies of the rotational relaxation of a rigid rod-like polymer in a concentrated solution, such a polymer is confined in a small cage made by the neighboring polymers and has a life time $\tau_{1}$ due to the Brownian motion. ${ }^{19-21}$ The observation on the time-scale longer than $\tau_{1}$ reveals the relaxation due to the end-over-end rotation of the rod-like polymer, while the observation within the time shorter than $\tau_{1}$ reveals the relaxation caused by the rotational diffusion in a limitted angle in the cage. ${ }^{21} \mathrm{We}$ assume that the present case of solid (PnALG)s is the special one in which the side chains play a role of the viscous solvent and the life time $\tau_{1}$ is infinitely long.

The rotational diffusion constant $D_{\mathrm{r}}$ of a rod-like polymer in a solution is given by the relation, ${ }^{20}$

$$
D_{\mathrm{r}}=\frac{3 k T}{\pi \eta_{\mathrm{s}} L^{3}} \log P
$$

where $\eta_{\mathrm{s}}, L$, and $P$ are the solvent viscosity, rod length and ratio of $L$ to the rod diameter $d$. Here, the end correction is neglected. On the other hand, the rotational relaxation time $\tau_{\mathrm{r}}$ in the cage is given by

$$
\tau_{\mathrm{r}}=\left\langle\theta^{2}\right\rangle / D_{\mathrm{r}}
$$

where $\left\langle\theta^{2}\right\rangle$ is the space average of the rotational angle $\theta$ squared. ${ }^{21}$

The strength of the dielectric relaxation due to the orientation polarization of the rod-like polymer can be estimated from the incremental polarizability due to the dipolar fluctuation by the relation, ${ }^{21}$

$$
\Delta \varepsilon=4 \pi \frac{N\left\langle\theta^{2}\right\rangle \mu^{2}}{2 k T}\left(\frac{E}{E_{\mathrm{e}}}\right)
$$

where $N, \mu, E$, and $E_{\mathrm{e}}$ are the number of rodlike polymers in unit volume, the dipole moment of the rod-like polymer along the long axis, the internal and external electric field strengths, respectively.

In the case of the $\alpha$-relaxation in PMLG, the data given in the previous paper ${ }^{12}$ show that the loss permittivity maximum appears at $c a$. $3 \times 10^{-6} \mathrm{~Hz}$ and $\Delta \varepsilon$ is $c a .2$ at $9^{\circ} \mathrm{C}$. The average molecular weight of the sample is $c a .142,000$. The molecular weight of the structural unit is 142 and thus the degree of polymerization is ca. $10^{3}$. The value of $L$ is then $c a .1 .5 \times 10^{3} \AA$, where $1.5 \AA$ is the length of the $\alpha$-helix per one residue. ${ }^{22}$ The value of $\theta$ is thought to be of the order of $a / L$, where $a$ is the diameter of the cage. The values of $a$ and $d$ are assumed to be $12 \mathrm{~A}$ and $8 \mathrm{~A}$ respectively from the data on the hexagonal structure of the $\alpha$-helices in solid (PnALG)s. ${ }^{8}$ Consequently, $\theta$ is of the order of $10^{-2} \mathrm{rad}$. Here, we assume that $\left\langle\theta^{2}\right\rangle=1 \times$ $10^{-4}$ or $\left(\left\langle\theta^{2}\right\rangle\right)^{1 / 2}=1 \times 10^{-2} \mathrm{rad} \doteqdot 0.6^{\circ}$.

The value of $\eta_{\mathrm{s}}$ is estimated as follows. Though the glass transition does not occur in the isoviscous state, the viscosity of polystyrene at $T_{\mathrm{g}}$ was of the order of $10^{11}$ poise for a rather wide range of molecular weight. $^{23}$ Therefore, we assume that $\eta_{\mathrm{s}}$ is also $c a .10^{11}$ poise at $T_{\mathrm{g}}$. As shown in the previous paper, $T_{\mathrm{g}}$ of PMLG is $c a .5^{\circ} \mathrm{C}$ and the difference in the logarithm of the shift factor between $5^{\circ} \mathrm{C}$ and $9^{\circ} \mathrm{C}$ is $c a$. 1 . Consequently, $\eta_{\mathrm{s}}$ at $9^{\circ} \mathrm{C}$ is $c a$. $10^{10}$ poise. 
If these values are used, the values of $D_{\mathrm{r}}$ and $\tau_{\mathrm{r}}$ in eq 3 and 4 are $c a .5 \times 10^{-9} \mathrm{~s}^{-1}$ and $2 \times 10^{4}$ s respectively. Therefore, the frequency for the loss-permittivity maximum, $f_{\mathrm{m}}=1 /\left(2 \pi \tau_{\mathrm{r}}\right)$, is $c a .8 \times 10^{-6} \mathrm{~Hz}$.

As for eq 5 , the value of $\mu$ is $c a$. $3.6 \times 10^{-18} \times 10^{3} \mathrm{esu}$, because the dipole moment along the helical axis is $3.6 \times 10^{-18}$ esu per one residue. ${ }^{22}$ As the density of PMLG at a room temperature is reported to be 1.31 $\mathrm{g} \mathrm{cm}^{-3,22} N$ is $c a .5 .5 \times 10^{18} \mathrm{~cm}^{-3}$. The value of $E / E_{\mathrm{e}}$ is assumed to be $c a .3$, because this is generally the case. Using these values, we get the value $c a .3$ for $\Delta \varepsilon$.

The values of $f_{\mathrm{m}}$ and $\Delta \varepsilon$ calculated above are in good agreement with the experimental results in the order of magnitude. As the values of the various quantities used for eq 4,5 , and 6 are not unreasonable, the explanation for the nature of the $\alpha$-relaxation presented here seems to be not unreasonable.

The nature of the $\alpha$-relaxation discussed above can explain the fact that the $\alpha$-relaxation and dc conduction process have the same shift factor, ${ }^{12}$ because the resistance to these two processes is thought to be given by the viscosity of the same liquid of the side chains.

\section{The Nature of the Glass Transition}

The simple model used to explain the mechanism of the $\alpha$-relaxation can be used also to explain the nature of the glass transition associated with the $\alpha$-relaxation. In this model, the motion of the rod-like molecule should be frozen as a result of the vitrification of the viscous liquid, obeying the WLF equation when the temperature is lowered enough. This behavior of the model corresponds to the glass transition mentioned above. Here, the rod-like molecule corresponds to the rigid core of the $\alpha$ helix, but the relation between the viscous liquid and the side chain should be considered in more detail.

It should be noted that the viscous liquid represents only the resistance to the motion of the rigid core of the $\alpha$-helix and that a trans- lational motion of the side chain occurs inevitably in connection with the motion of the core of the $\alpha$-helix to which it is attached. Therefore, the resistance to the motion of the core of the $\alpha$-helix is caused by the resistance to such motion of the side chain. The resistance in this case is due to the interaction between the side chains of the neighboring helices in such motion and its temperature dependence should be represented by the WLF equation as in agreement with the experimental result. The translational motion of the side chain is frozen together with the motion of the core of the $\alpha$ helix when the viscous resistance becomes high enough.

However, the internal rotation of the side chain is not frozen even below the glass transition temperature and causes the $\beta$-relaxation. The resistance to this process is represented by the Arrhenius equation as a function of temperature. Therefore, if the explanation given above is correct, the glass transition observed here is essentially due to the freezing of the motion of the rigid $\alpha$-helix.

Further investigation of the validity of the explanation for the $\alpha$-relaxation and the glass transition, a study of the relation between the dielectric and mechanical relaxations and a study using samples with longer side chains will be conducted in the near future.

\section{REFERENCES}

1. Y. Hashino, M. Yoshino, and K. Nagamatsu, Rep. Prog. Polym. Phys. Jpn., 9. 297 (1966).

2. Y. Yokomori, Y. Uematsu, and I. Uematsu, Rep. Prog. Polym. Phys. Jpn., 15, 633 (1972).

3. A. Tsutsumi, K. Hikichi, T. Takahashi, Y. Yamashita, M. Matsushima, M. Kanke, and M. Kaneko, J. Macromol. Sci. Phys., 8, 413 (1973).

4. A. Tanaka and Y. Ishida, J. Polym. Sci., Polym. Phys. Ed., 11, 1117 (1973).

5. A. L. Nguyen, B. T. Vu, and G. L. Wilkes, J. Macromol. Sci. Phys., 9, 367 (1974).

6. Y. Yamashita, A. Tsutsumi, K. Hikichi, and M. Kaneko, Polym. J., 8, 114 (1975).

7. K. Hikichi, A. Tsutsumi, S. Isozaki, and M. Kaneko, Polym. J., 7, 646 (1975).

8. N. Matsushita and K. Hikichi, Polym. J., 10, 437 
(1978).

9. N. Sasaki, H. Shimodate, Y. Yamashita, and K. Hikichi, Polym. J., 11, 983 (1979).

10. Y. Yamashita, K. Hikichi, and M. Kaneko, Polym. J., 12, 439 (1980).

11. N. G. McCrum, B. E. Read and G. Williams, "Anelastic and Dielectric Effects in Polymeric Solids," Wiley, New York, N. Y., 1967.

12. M. Kakizaki, H. Nakayama, H. Tanaka, and T. Hideshima, Polym. J., 17, 1179 (1985).

13. J. D. Ferry, "Viscoelastic Properties of Polymers," 3rd ed, Wiley, New York, N. Y., 1980.

14. C. J. F. Böttcher and P. Bordewijk, "The Theory of Electric Polarization," Vol. II, 2nd ed, Elsevier, Amsterdam, 1978.

15. B. V. Hamon, Proceedings of Instr. Electr. and
Engineering, Part 4, 99, 151 (1952).

16. T. Tetsutani, M. Kakizaki, and T. Hideshima, Polym. J., 14, 305 (1982).

17. Y. Ishida and K. Yamafuji, Kolloid. Z., 177, 97 (1961).

18. R. Hayakawa and Y. Wada, J. Macromol. Sci., Phys., 8, 445 (1973).

19. M. Doi, J. Phys. (Paris), 36, 607 (1975).

20. H. Nakajima and Y. Wada, Biopolymers, 16, 875 (1977).

21. Y. Mori, N. Ookubo, R. Hayakawa, and Y. Wada, J. Polym. Sci., Polym. Phys. Ed., 20, 2111 (1982).

22. R. Pethig, "Dielectric and Electronic Properties of Biological Materials," Wiley, New York, N. Y., 1979.

23. T. G. Fox and P. J. Flory, J. Appl. Phys., 21, 581 (1950). 